\title{
Stripe pattern in the intensity profile of collimated soft x-ray beams caused by surface corrugation of the refocusing mirrors
}

\author{
Detlef Schmitz*, Frank Siewert, Thomas Zeschke
}

December 9, 2014

\begin{abstract}
Helmholtz-Zentrum Berlin für Materialien und Energie, Albert-Einstein-Straße 15, D-12489 Berlin, Germany
\end{abstract}

\begin{abstract}
The effect of progress in surface finishing of optical components on the collimated-beam properties of soft x-ray beamlines at synchrotron radiation facilities is demonstrated: a stripe pattern, experimentally observed in the $2 \mathrm{D}$ intensity profile of beamlines with optical components manufactured 10-15 years ago, would be strongly attenuated if the existing refocusing mirror was replaced by an ultra-precise mirror manufactured with state-of-the-art of today surface finishing techniques. The observed stripe pattern is not caused by diffraction because its period length did not change with photon energy. Instead it can be explained with geometrical optics and is due to the height profile of the refocusing mirror which has been independently measured with a long trace profiler and used as an input in our raytracing simulations.
\end{abstract}

*corresponding author (schmitz@helmholtz-berlin.de) 


\section{Introduction}

Synchrotron radiation in the soft $\mathrm{x}$-ray range from electron storage rings is prepared for scientific experiments by beamlines using mirrors and gratings at grazing incidence. The surface quality of these optical components is essential for the imaging properties and the energy resolution of the beamlines. During the last 10-15 years the residual slope errors of these optical components has been significantly reduced by about one order of magnitude [1]. Up-to-date mirrors exhibit residual slope error values in the range of a few $0.1 \mu \mathrm{rad}$ depending on the shape of the surface [1]. This has been achieved by improvements in the surface finishing technologies, i.e. ion beam figuring $[2,3]$ and elastic emission machining [4], and the slope error metrology. The corresponding achievable focus sizes strongly decreased as demonstrated e.g. at BESSY II [5] and the Diamond Light Source [6].

Dedicated instrumentation has been established in the synchrotron community to measure the topography of optical elements like mirrors. The state-of-the-art of today Nanometer Optical component measuring Machine (NOM) at BESSY II [7] reaches sub-nm accuracy $[8,9]$ and is a calibrated instrument [10]. In terms of slope error it provides an accuracy in the range of some 10 nrad random mean square (rms) [8, 9] whereas the classical Long Trace Profiler (LTP)-II used in the past at BESSY II to inspect the here discussed optics allowed to measure optical components with an uncertainty of $500 \mathrm{nrad}$ rms. The LTP was based on the principle of a pencil-beam interferometer [11]. The measurement beam (a double beam) was traced along the line of inspection by moving the optics-head on an air-bearing based carriage and generated an interference pattern. Slope and height profiles were extracted from a sequence of interference patterns [12]. The LTP-II design used in the past at BESSY II followed the idea of a scanning optics-head with an additional reference beam to suppress the impact of guiding errors (mainly the pitch error) contributing to the error budget of the instrument. As has first been shown by S. Qian et al. at Elettra, this concept had significant drawbacks compared to the scanning penta-prism design [13]. Most state-of-the-art of today instruments like the NOM [8] operated at BESSY II as well as the upgraded LTPs at the ESRF [14] and Spring-8 [15] follow the latter design but use a $45^{\circ}$ double mirror setup instead of a bulk penta-prism.

Stripe patterns were observed out of focus at several beamlines at third generation synchrotron radiation facilities. At SOLEIL they were explained by the slope errors of the focusing mirrors in a Kirkpatrick-Baez configuration using a geometrical approach. They were measured for a photon energy of $140 \mathrm{eV}$ but not studied as a function of photon energy [16]. In the hard x-ray range beamlets were observed and deflected into the main focus by bending also the ends of the focusing mirror to the right curvature [6].

In the present paper we report on the stripe pattern observed in the intensity profile of the collimated beam (see Fig. 1) produced by a soft x-ray plane grating monochromator (PGM) beamline at the electron storage ring BESSY II [17]. The optical design of this type of beamlines was introduced by R. Follath and F. Senf [18]. In short, the optical design (see Fig. 2) is as follows: In the vertical 
plane, the toroidal mirror M1 creates a collimated beam for the monochromator which consists of the vertically deflecting plane mirror M2 and the plane grating G. Behind the monochromator the beam is focused by the cylindrical mirror M3 to the exit slit. Finally the beam is typically refocused by the toroidal mirror M4 (case A). In the horizontal plane, mirror M1 creates an intermediate focus $1.5 \mathrm{~m}$ upstream of the exit slit and the refocusing is analogous to the vertical plane. This astigmatic intermediate focusing has the advantage that the horizontal size of the focus located $1 \mathrm{~m}$ downstream of M4 (case A) is reduced due to the increased entrance arm length of M4.

One special feature of the beamline shown in Fig. 2 is that for experiments one can choose between a focused (case A) and a collimated beam (case B) by using the appropriate last mirror [19]. The collimated beam is advantageous regarding the flexibility in the distance of the endstations from the last mirror and with regard to measuring samples which are sensitive to radiation damage with low photon flux density.

Here we concentrate on the intensity profile of the collimated beam which exhibits a pattern consisting of vertical stripes. This stripe pattern (Fig. 1) was observed experimentally and reproduced with raytracing simulations. The height profiles of the mirrors, which were measured as part of the quality control, were used as an input in the raytracing simulations. The manufacturing of the mirrors, the measurement of their height profiles and the measurement of the stripe pattern took place during the construction and commissioning of the beamline already in the years 2001 and 2002. Now we revisited these results in order to explain the observed stripe pattern and to demonstrate the impact of the progress in the surface finishing of grazing incidence optical components used in synchrotron radiation beamlines during the last 10-15 years with an outstanding example: according to our raytracing simulations the stripe pattern in the intensity profile would be strongly attenuated if ultra-precise up-to-date mirrors were used. This clearly demonstrates the potential improvement of the performance of existing beamlines and upcoming beamline projects.

\section{Intensity profile measurements}

The particular intensity profiles discussed here were measured during the initial commissioning of a PGM beamline in spring 2002. During this commissioning a fine alignment of all optical components in the beamline was performed with synchrotron radiation. The last mirrors $\mathrm{M} 4 \mathrm{a}$ and $\mathrm{M} 4 \mathrm{~b}$ were aligned by imaging and optimizing the shape of the focused (Fig. 2, case A) and collimated beam (Fig. 2, case B), respectively. For the imaging a combination of a fluorescent screen, a zoom lens and a CCD camera was used. The fluorescent screen consists of a quartz disk which is coated with high-resolution phosphor in order to convert the soft $\mathrm{x}$-rays to the visible spectral range. At the same time the quartz disk serves as the vacuum-air interface. The images of the beam are detected via a zoom lens with a CCD camera, both located in ambient air. Fluorescent screen, zoom lens and CCD camera are mounted on a carriage which can be aligned 
with and translated along the beam direction [20].

The measured 2D intensity profile of the collimated beam and a horizontal line scan are shown in Fig. 1. A pattern mainly consisting of vertical stripes is clearly visible. This is the first main result. The stripe pattern moved horizontally and was otherwise unaltered when M4b was rotated around the vertical axis. It clearly changed when M4b was translated horizontally so that different parts of the mirror surface were illuminated. In this case the pattern changed in a way as if different parts along the horizontal direction of a stationary stripe pattern were illuminated. So the second main result is that the stripe pattern clearly depended on which part of the optically used area of M4b was exposed to the beam. The intensity profile shown in Fig. 1 was measured at a photon energy of $402 \mathrm{eV}$. If the stripe pattern was due to diffraction, then its period length would decrease with increasing photon energy. Stripe patterns measured at photon energies of $700 \mathrm{eV}, 1000 \mathrm{eV}, 1250 \mathrm{eV}$ and $1450 \mathrm{eV}$ are shown in Figs. $3 \mathrm{a}, \mathrm{b}, \mathrm{c}$ and $\mathrm{d}$, respectively. The corresponding linescans, compared in Fig. 3e, clearly demonstrate that the period length of the stripe patterns did not change with the photon energy which has been varied by more than a factor of two. Therefore, the stripe patterns cannot be due to diffraction. This is the third main result. The stripe pattern can very well be explained with geometric optics and is caused by the height profile of M4b as depicted in the following sections. In the focused case (Fig. 2, case A) no stripes have been observed, neither in the focus nor in a range of $\pm 0.15 \mathrm{~m}$ around the focal distance of mirror M4a.

\section{$3 \quad$ Height profile measurements}

The height profiles of the mirrors were measured with the LTP-II at BESSY II as part of the quality control before their final mounting in the beamline. Typical height deviation line scans measured along the long axes of the horizontally deflecting mirrors M1, M3 and M4b are shown in Fig. 4 as black, red and blue lines, respectively. The height deviations from segments of circles with fitted radii in the meridional planes are shown. The corresponding rms values of the height deviations and the corresponding slope errors are summarized in Tab. 1. The values of a hypothetical mirror M4 explained in the next section are also given in Tab. 1. The mirrors M1, M3 and M4b were manufactured during the year 2001 and their slope error values are typical for mirrors produced in that time period. The measured height profiles were used as an input in the raytracing simulations as described in the following section.

\section{Raytracing simulations}

In order to find an explanation for the stripe pattern observed in the collimated beam, the beamline optics was simulated with the raytracing program RAY [21]. Independently measured height profiles of the horizontally deflecting mirrors M1, M3 and M4b were used as an input. Each height profile consisted of 
six linescans along the long axis of the mirror with lateral distances of $2 \mathrm{~mm}$. A stripe pattern was obtained only after increasing the height deviations of M4b by a factor of 1.5. This superelevation can be justified by an underestimation of the height deviation amplitude in the LTP-II measurements at BESSY II as discussed in the next section. The height deviations of M3 were also multiplied by a factor of 1.5 for the sake of consistency and this did not affect the stripe pattern but rather the long-wave background. The height deviations of M1 were not multiplied by a factor because its typical lateral wavelength of the corrugation is much larger and the uncertainty in the LTP-II measurements smaller than for M3 and M4b. The resulting vertically integrated simulated stripe pattern is shown in Fig. 5 (red line) and compared with the linescan of the measured stripe pattern (black line). The period length and the intensity modulation amplitude of the measured stripe pattern are well reproduced in the simulation using the height profile of M4b.

In order to demonstrate the effect of reducing the residual slope error on the stripe pattern, we replaced M4b by a hypothetical ultra-precise mirror in the simulations. For the hypothetical mirror the radii of $\mathrm{M} 4 \mathrm{~b}$ have been combined with the height deviations of an existing ultra-precise mirror polished with stateof-the-art of today techniques resulting in a residual slope error of only $0.13 \mu \mathrm{rad}$ rms (see last line in Tab. 1). With this hypothetical ultra-precise mirror the stripe pattern is strongly attenuated as shown in Fig. 5 as a blue line.

A slowly varying background in the intensity profile caused by long-wave corrugations of the mirror surfaces remains. The increase in the background of the simulations on the left side, i.e. for positions between $0.5 \mathrm{~mm}$ and $0.75 \mathrm{~mm}$, is mainly caused by the height profile of M1 because it moves towards the right side if the orientation of M1 is reversed along its long axis. Reversing the orientation along the long axes of the height profiles of M1 and M3 in the simulations changed only the long wave background but not the stripe pattern. The small variation of the intensity from point to point with a position stepwidth of 12 $\mu \mathrm{m}$ is due to statistical noise and does not depend on the superelevation factor of the height profiles.

We conclude that the experimentally observed stripe pattern can be described with geometrical optics, is mainly due to the height profile of the last mirror M4b and would be strongly attenuated if M4b was replaced by an ultra-precise state-of-the-art of today mirror.

\section{Discussion}

The stripe pattern observed in the intensity profile of the collimated beam is not caused by diffraction because its period length did not change with the photon energy which was varied by more than a factor of 3 in the experiment. The reasons for this result are the small transversal coherence length of the undulator beam entering the beamline compared with the mirror dimensions and the fact that the optical path differences induced by the height deviations at grazing incidence are small compared with the wavelength of the soft x-rays. 
The transversal coherence length is $345 \mu \mathrm{m}(96 \mu \mathrm{m})$ for a photon energy of 402 $\mathrm{eV}(1450 \mathrm{eV})$ at the distance of the first mirror from the undulator, i.e. $17 \mathrm{~m}$. The rms height deviation of M4b of $3.9 \mathrm{~nm}$ (see Tab. 1) induces at a grazing incidence angle of 1.8 degrees a rms pathlength difference of $0.12 \mathrm{~nm}$ which amounts to $1 / 25$ of the wavelength at a photon energy of $402 \mathrm{eV}$. At this photon energy the reflection at M4b is diffraction-limited because it clearly fulfills the Maréchal criterion [22] according to which the induced rms phase error of the wavefront may be at most $1 / 14$ of its wavelength. Only at photon energies above $724 \mathrm{eV}$ the reflection at M4b is no longer diffraction-limited. Nevertheless, the period length of the stripe pattern did not change even for the highest measured photon energy of $1450 \mathrm{eV}$. Therefore the stripe pattern cannot be caused by diffraction. The observed stripe pattern can rather be explained by geometrical optics and is mainly due to the height profile of the last mirror M4b of the beamline. This result is in accordance with the geometrical approach of T. Moreno et al. [16].

The other horizontally deflecting mirrors M1 and M3 cause a rather long-wave modulation of the background as verified in our raytracing simulations by reversing the position of their height profiles along the long axis (not shown). Spatial period lengths of the figure errors of more than $20 \mathrm{~mm}$ as present on mirror M1 can be corrected by a bimorph piezoelectric mirror in combination with the pencil-beam method as demonstrated by J. Sutter et al. [23]. The major spatial period lengths of mirror M4b could not be corrected by a bimorph mirror because they are below $20 \mathrm{~mm}$.

In our raytracing simulations the amplitude of the height deviations had to be increased by a factor of 1.5 in order to obtain a stripe pattern in the intensity profile of the collimated beam as observed in the experiment. This finding indicates that the amplitude of the height deviations was underestimated in the LTP-II measurements. The above-mentioned factor of 1.5 is in accordance with the experimental error of the slope error measurements of $0.5 \mu \mathrm{rad}$ rms. This relatively large error was mainly caused by systematic errors of the LTP-II at BESSY II [13] and by random instabilities of the environment [24] during the years 2001/2002 at BESSY II.

According to our raytracing simulations the stripe pattern would be strongly attenuated if the collimating mirror M4b was replaced by an ultra-precise mirror polished with state-of-the-art of today techniques. A slowly varying background in the intensity profile caused by long-wave corrugations of the mirror surfaces would remain. Overall, the intensity profile of the resulting collimated beam would be much more homogeneous.

\section{Acknowledgments}

We would like to thank H. Lammert and H.H. Rossner for their support with the measurements, R. Follath and F. Senf for fruitful discussions, F. Schäfers for his support with the raytracing simulations and R. Follath for his critical reading of the paper. 


\section{References}

[1] F. Siewert, Journal of Physics: Conference Series 425 (2013) 152001.

[2] A. Schindler, T. Hänsel, A. Nickel, H. Lammert, F. Siewert, Optical Manufacturing and Testing V, Book Series: Proc. of SPIE 5180 (2003) 64-72.

[3] H. Thiess, H. Lasser, F. Siewert, Nucl. Instr. and Meth. in Physics Research A 616 (2010) 157-161.

[4] K. Yamauchi, H. Mimura, K. Inagaki, Y. Mori, Rev. Sci. Instrum. 73 (2002) 11.

[5] F. Siewert, H. Lammert, T. Noll, T. Schlegel, T. Zeschke, T. Hänsel, A. Nickel, A. Schindler, B. Grubert, C. Schlewitt, Proc. of SPIE 5921 (2005) 592101.

[6] J. Sutter, S. Alcock, K. Sawhney, J. Synchrotron Rad. 19 (2012) 960-968.

[7] F. Siewert, J. Buchheim, T. Zeschke, G. Brenner, S. Kapitzki, K. Tiedtke, Nucl. Instr. and Meth. in Physics Research A 635 (2011) S52-S57.

[8] F. Siewert, J. Buchheim, S. Boutet, G. J. Williams, P. A. Montanez, J. Krzywinski, R. Signorato, Optics Express 20 (2012) 4526.

[9] F. Siewert, J. Buchheim, T. Zeschke, J. Synchrotron Radiation 21 (2014) 968-975.

[10] F. Siewert, J. Buchheim, T. Zeschke, Nucl. Instr. and Meth. in Physics Research A 616 (2010) 119-127.

[11] P. Takacs, S. Qian, J. Colbert, Proc. of SPIE 749 (1987) 59-64.

[12] S. C. Irick, Rev. Sci. Instrum. 63 (1992) 1432-1435.

[13] S. Qian, W. Jark, P. Z. Takacs, Rev. Sci. Instrum. 66 (1995) 2562.

[14] A. Rommeveaux, L. Assoufid, H. Ohashi, H. Mimura, K. Yamauchi, J. Qian, T. Ishikawa, C. Morawe, A. Macrander, A. Khounsary, S. Goto, Proc. of SPIE 6704 (2007) 67040B.

[15] Y. Senba, H. Kishimoto, H. Ohashi, H. Yumoto, T. Zeschke, F. Siewert, S. Goto, T. Ishikawa, Nucl. Instr. and Meth. in Physics Research A 616 (2009) S237-S240.

[16] T. Moreno, R. Belkhou, G. Cauchon, M. Idir, Proc. of SPIE 5921 (2005) $59210 \mathrm{~F}$.

[17] D. Schmitz, H. Rossner, P. Imperia, H. Maletta, J. Bahrdt, R. Follath, W. Frentrup, A. Gaupp, K. Holldack, H.-C. Mertins, F. Senf, S. Valencia, BESSY Annual Report (2002) 358-361. 
[18] R. Follath, F. Senf, Nucl. Instr. and Meth. in Physics Research A 390 (1997) 388-394.

[19] U. Englisch, H. H. Rossner, H. Maletta, J. Bahrdt, S. Sasaki, F. Senf, K. J. S. Sawhney, W. Gudat, Nucl. Instr. and Meth. in Physics Research A 467 (2001) 541-544.

[20] K. Holldack, T. Zeschke, F. Senf, C. Jung, R. Follath, D. Ponwitz, BESSY Annual Report (2000) 336.

[21] F. Schäfers, In: Springer Series in Modern Optical Sciences: Modern Developments in X-Ray and Neutron Optics, eds. A. Erko, M. Idir, Th. Krist, A. G. Michette, Springer Berlin/Heidelberg 137 (2008) 9-41.

[22] A. Maréchal, Revue d'Optique, Theorique et Instrumentale 26 (1947) 257.

[23] J. P. Sutter, S. G. Alcock, K. J. S. Sawhney, Proc. of SPIE 8139 (2011) 813906.

[24] H. Lammert, F. Senf, M. Berger, Proc. of SPIE 3152 (1997) 168-179. 
Table 1: Values for the height deviations and slope errors of the horizontally deflecting mirrors in the beamline (M1, M3, M4b) and an ultra-precise hypothetical collimating mirror produced with state-of-the-art finishing techniques.

\begin{tabular}{|c|c|c|c|}
\hline mirror & shape & $\begin{array}{c}\text { rms height deviation } \\
(\mathrm{nm})\end{array}$ & $\begin{array}{c}\text { rms slope error } \\
(\mu \mathrm{rad})\end{array}$ \\
\hline M1 & toroid & 9.8 & 1.24 \\
M3 & cylinder & 2.1 & 0.63 \\
M4b & toroid & 3.9 & 1.46 \\
\hline M4 hypoth. & toroid & 0.56 & 0.13 \\
\hline
\end{tabular}




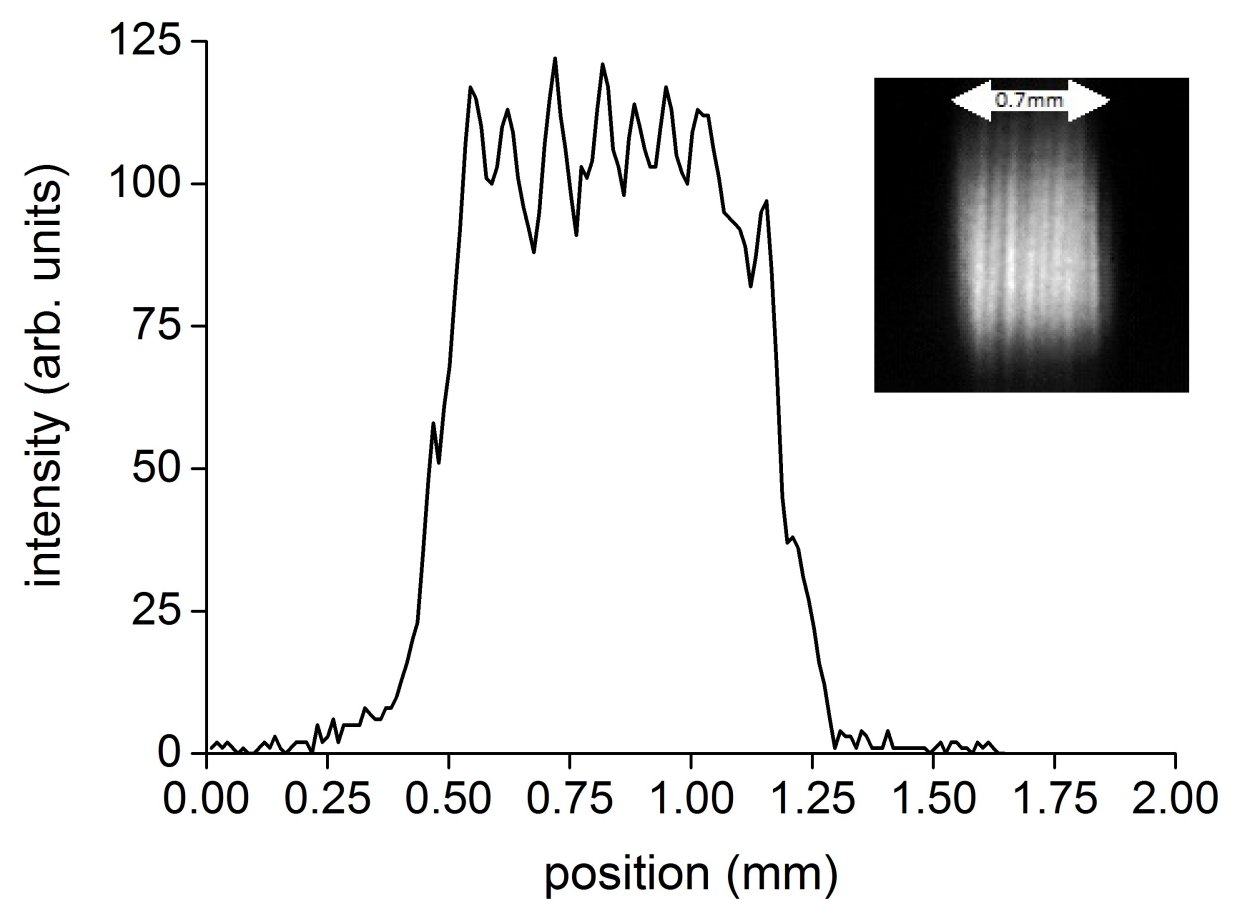

Figure 1: Horizontal intensity linescan of the 2D intensity profile (inset) of the collimated beam measured at a photon energy of $402 \mathrm{eV}$. 

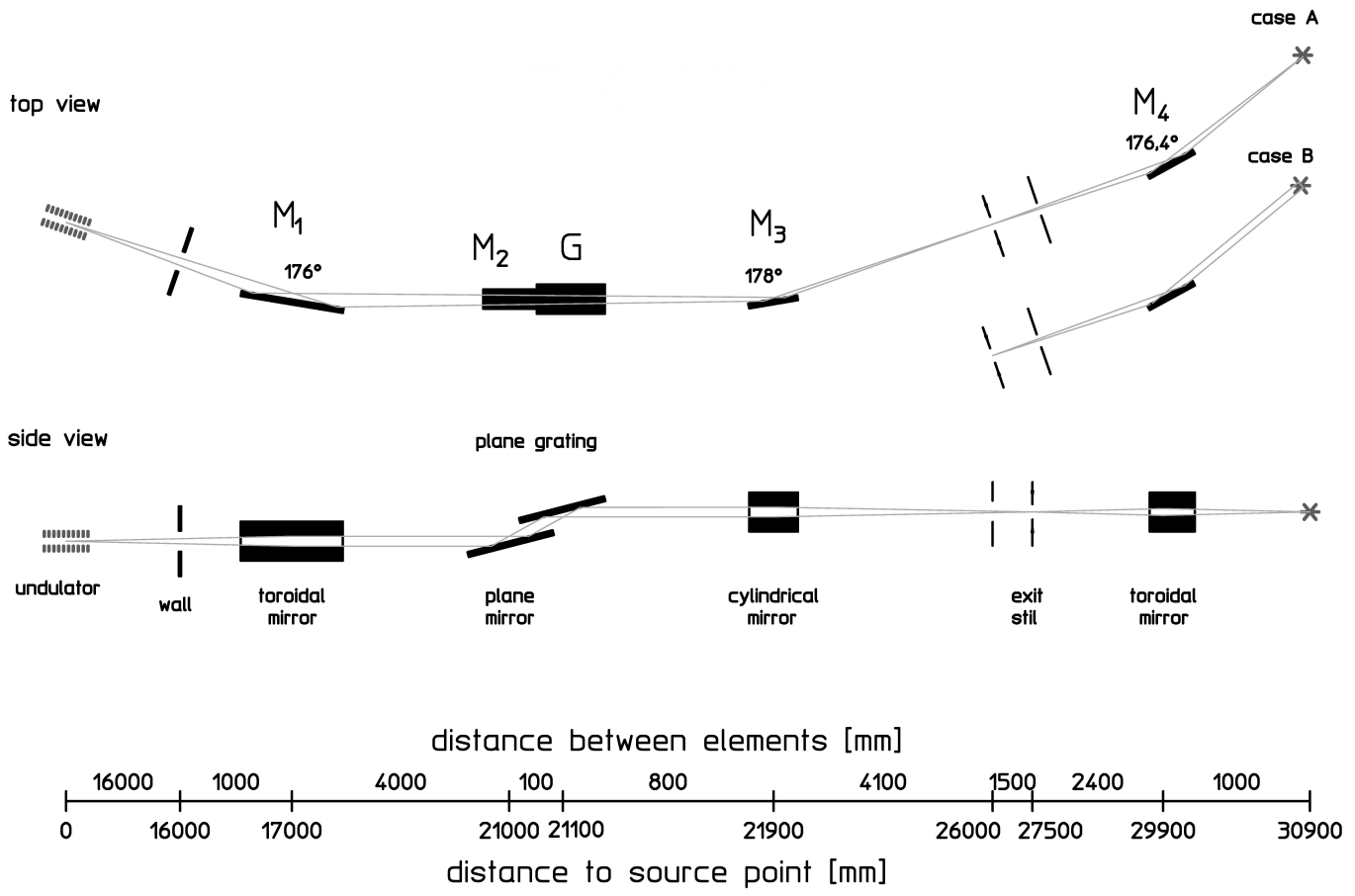

Figure 2: Optical layout of a plane grating monochromator beamline [19]. At the sample position on the right a focused (case A) or a collimated beam (case B) can be chosen. 

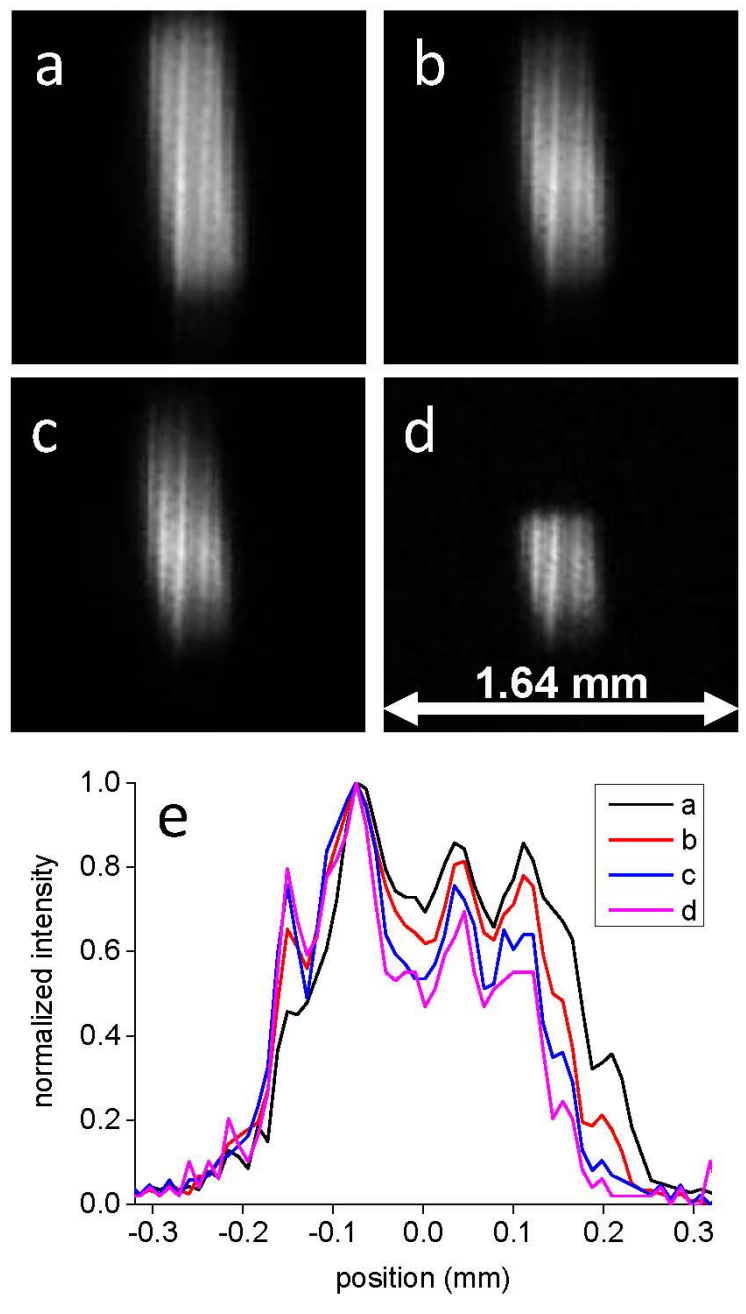

Figure 3: 2D intensity profiles of the collimated beam measured at photon energies of $700 \mathrm{eV}$ (a), $1000 \mathrm{eV}$ (b), $1250 \mathrm{eV}$ (c) and $1450 \mathrm{eV}$ (d). The corresponding normalized linescans (e) demonstrate that the period length of the stripe patterns does not change with the photon energy. The vertical beam size decreases with increasing photon energy due to the decreasing grating angle, i.e. the angle between the surface of the grating and the horizontal. 


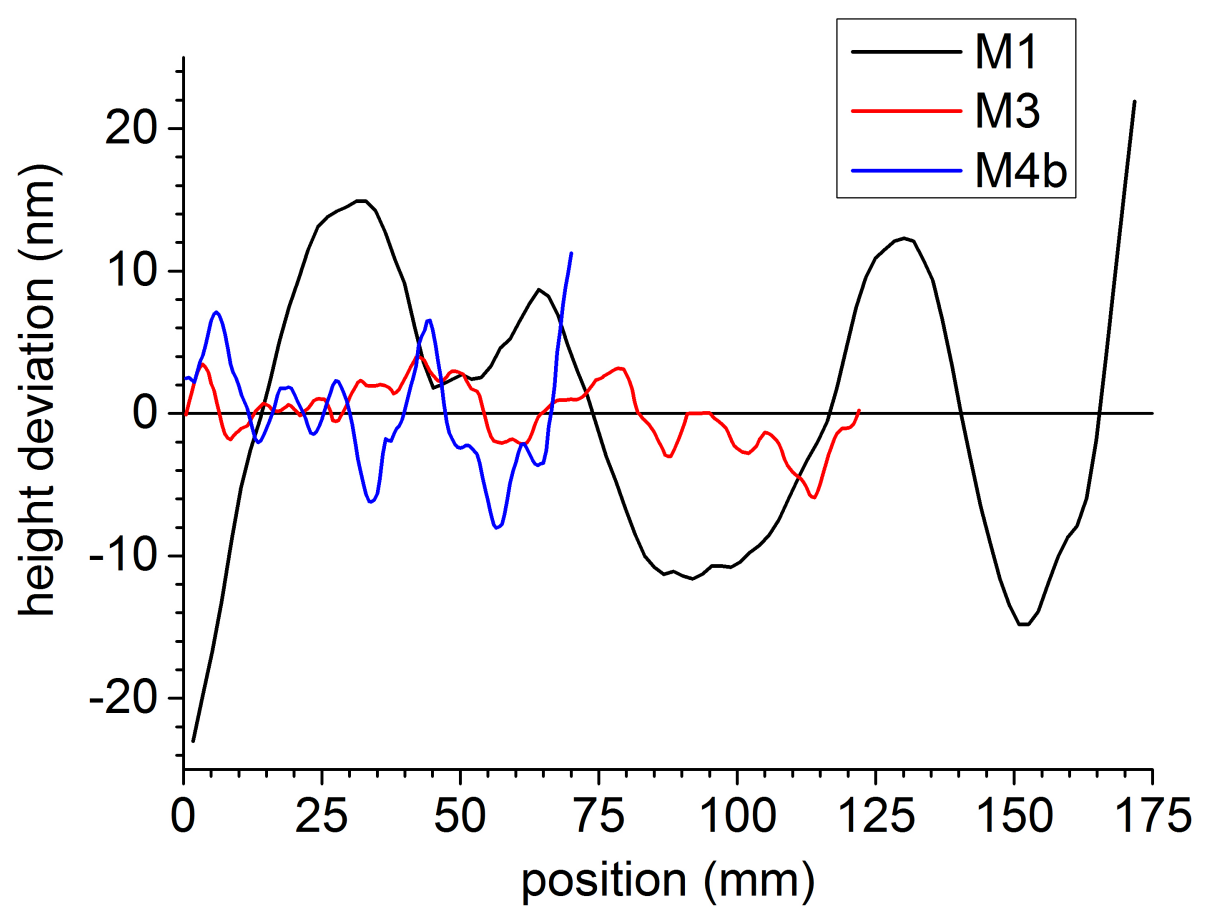

Figure 4: Height deviations along the long axes of the horizontally deflecting mirrors M1, M3 and M4b. 

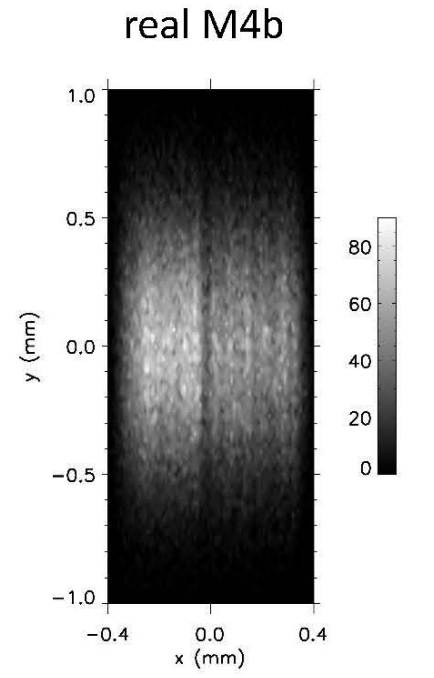

\section{hypothetical M4}
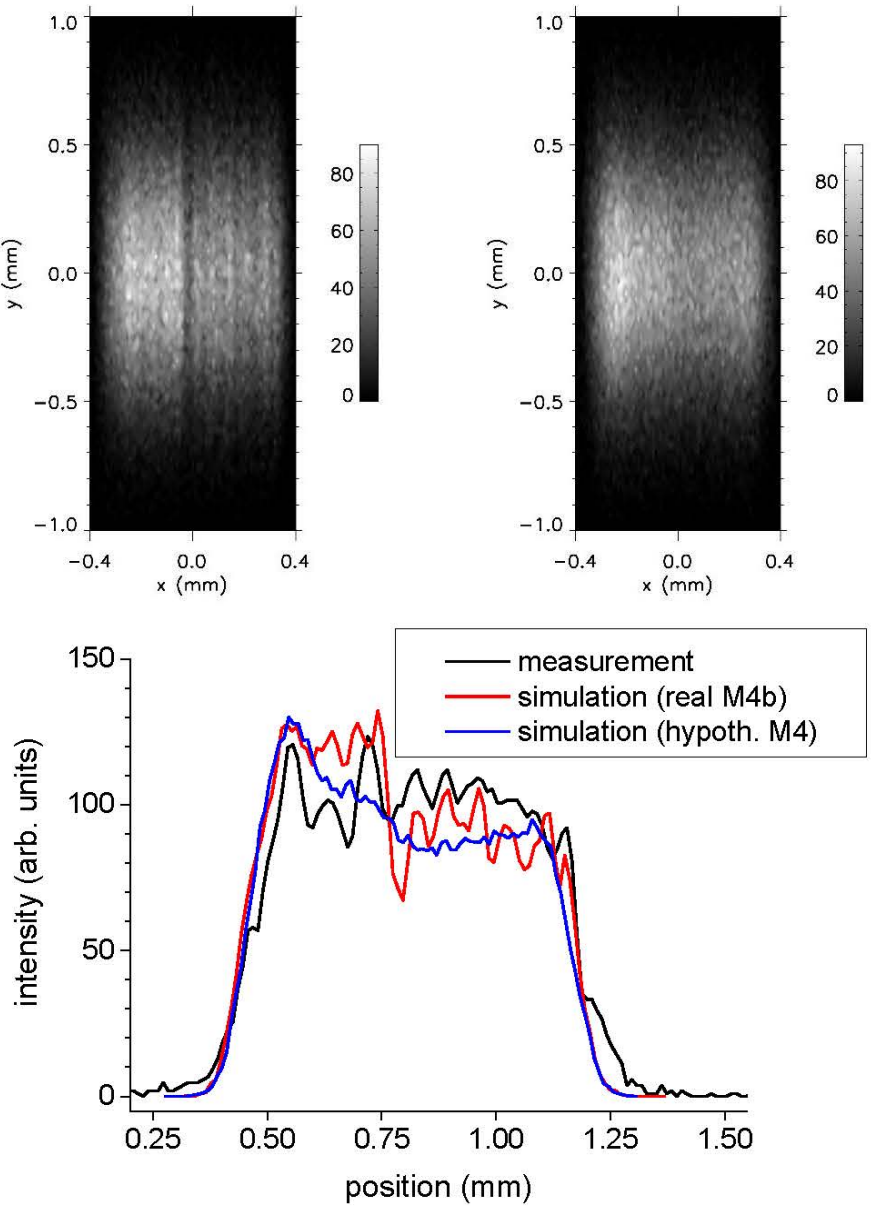

Figure 5: Upper panel: 2D intensity profiles obtained with raytracing using the real height deviations of M4b multiplied with 1.5 (left picture) and the height deviations of an existing ultra-precise mirror (right picture). Lower panel: Intensity linescans of the collimated beam obtained by measurement (black line) and by raytracing simulations, i.e. of the $2 \mathrm{D}$ intensity profiles shown in the upper panel on the left (red line) and on the right (blue line). 